\title{
Visual binding in the standing wave illusion
}

\author{
JAMES T. ENNS \\ University of British Columbia, Vancouver, British Columbia, Canada
}

\begin{abstract}
When two video frames are alternated at the appropriate rate, one with a central bar and the other with two flanking bars, the central bar becomes invisible. Competing explanations for this standing wave illusion are examined, with the results showing an influence of higher level shape representations on lower level edge processes. In Experiment 1, flanking bar duration was found to be more important to masking than central bar duration. Experiment 2 showed strong nonlocal effects in that masking of the central bar depended critically on whether it appeared in the same video frame as other visible bars. Experiments 3 and 4 showed that the contour shared by bars in separate frames was a less important factor than shape and surface similarity. This illusion is therefore an excellent tool for studying recursive interactions between higher level object representations and lower level contour processes.
\end{abstract}

Standing wave masking is an illusion in which a flickering target bar is rendered invisible by two counterphase flickering bars that flank the target (Macknik \& Haglund, 1999; Macknik \& Livingstone, 1998; Macknik, MartinezConde, \& Haglund, 2000). The illusion is sensitive to the spatial proximity of the bars in such a way that the target becomes increasingly more visible as the gap between the edges of the target and flanking bars is increased. The illusion is thus in many ways similar to classical metacontrast masking (Breitmeyer, 1984). However, an important difference is that the bars cycle continuously, leaving open the possibility of not only backward masking, as in classical metacontrast, but also forward masking. Standing wave masking also has the advantage of giving rise to percepts that have both continuously visible and invisible aspects. As such, the phenomenology of this form of masking contrasts sharply with the fleeting percepts associated with classical two-frame displays of metacontrast.

The present study is the first psychophysical exploration of the standing wave illusion. In the paper in which this form of masking was first reported, the illusion was presented as a demonstration, based on the study of classical two-frame masking displays (Macknik \& Livingstone, 1998). In the same paper, electrophysiologicalrecordings of standing wave displays were made in awake and anesthetized monkeys, and these indicated that single neurons in Area V1 are susceptible to the illusion. In a second paper, optical imaging of Area V1 confirmed that cycling displays of a target bar $(50 \mathrm{msec})$ and masking bars $(100 \mathrm{msec})$ resulted in suppression of the target bar representation (Macknik \& Haglund, 1999). Finally, a recent paper presented two psychophysical experiments as being relevant to the standing wave illusion, but again the experiments

Correspondence should be addressed to J. T. Enns, Department of Psychology, University of British Columbia, 2136 West Mall, Vancouver, BC, V6T 1Z4 Canada (e-mail: jenns@psych.ubc.ca). were based entirely on two-frame displays (Macknik et al., 2000).

Given this electrophysiological and optical imaging evidence for standing wave masking in neurons of Area V1, it is not surprising that the mechanisms proposed for the illusion are local inhibitory interactions between the transient neuronal signals associated with the onsets and offsets of the flickering bars. Specifically, it has been proposed that the critical temporal interval is about $100 \mathrm{msec}$ of elapsed time between offsets of the flickering bars (Macknik \& Livingstone, 1998) and that the strongest masking occurs at the leading and trailing temporal edges of the masking bars (Macknik et al., 2000). Yet, it is important that no systematic studies of the psychophysics of standing wave masking have yet been reported.

This study asked whether the standing wave illusion is determined primarily by local spatiotemporal interactions or whether higher level processes of shape and surface perception are also involved. The immediate motivation derives from the recently proposed object substitution theory of visual masking (Di Lollo, Enns, \& Rensink, 2000; Enns \& Di Lollo, 2000). This theory proposes that masking is a consequence of the ongoing recurrent communication between neurons at lower and higher levels of processing. Initial sensory input activates the spatially local and geometrically simple receptive fields of lower level units, which, in a feedforward sweep, activate units at higher levels that are sensitive over larger regions of the visual field and are tuned to more complex properties. In order to resolve ambiguity between alternative pattern activations at the higher level, and in order to bind patterns at the higher level to specific spatiotemporal locations, a feedback sweep of processing is required. Pattern hypotheses generated at the higher level are compared with the ongoing activity at the lower level. If the visual image remains stable over the iterations required to match the contents of these two levels to some criterion, conscious perception of the stimulus will ensue. However, if the input activity is altered before these iterations 
are complete, a mismatch will be detected and the iterative processes will begin again, this time based only on the sensory input that is currently activating the lower level neurons.

Three key predictions follow for the standing wave illusion:

1. Mask duration will be a more important predictor of masking effectiveness than either target duration or targetmask asynchrony. This prediction follows from the proposal that conscious object perception depends on some period of stability in the input, allowing the iterative processing loops to converge onto a single perceptual hypothesis. Extending the exposure duration of the mask makes it more likely that its representation will form the basis for perception.

2. Masking will be determined by visual properties that extend over larger regions than the receptive fields of neurons needed to register the edges of the stimuli. This is because processing iterations involve communication between regions of the brain that represent surface and object properties (but without a high degree of spatial precision) and regions that represent local edges with a high degree of spatial precision (but do not represent spatially extended properties such as shape, color, and texture).

3 . Everything else being equal, visually similar targets and flankers will result in stronger masking than will dissimilar items. This is because the iterative feedback operations attempt to confirm feature hypotheses activated by the initial feedforward sweep. Similar features in both phases of the iteration will therefore result in a better overall match, although the correctly identified features may be spatially mislocalized if the feedback sweep establishes their location to be different from the initial locations that activated these hypotheses.

Demonstrations of the effects in each of the following experiments can be seen on the Internet at www.interchange. ubc.ca/vsearch/standingwave/.

\section{GENERAL METHOD}

In each experiment, 10-12 naive observers rated the visibility of the target bar in each flickering display on a 7-point scale, $(7=$ target maximally visible, $1=$ invisible target). Visibility ratings were anchored at the upper end in each experiment by including conditions in which the target bar was at least as visible as the flanking bars (e.g., flanking bar duration of $17 \mathrm{msec}$ ).

Visibility ratings were used instead of forced-choice discrimination (e.g., discriminating which of two target bars is longer) because in pilot tests, target bars that were shorter than the flanking bars influenced the apparent length of the flanking bars. That is, observers were able to discriminate the flickering pattern that contained the shorter target bar quite accurately, not by seeing the target bars themselves, but by monitoring the illusory length changes in the flickering flanking bars. The flanking bars appear to shrink and expand with each display cycle, as though the short length of the unseen target were being attributed to the seen flanking bars. Similar observations have been reported previously with other types of displays (Herzog \& Koch, 2001; Wilson \& Johnson, 1985). It is notable that theory based on local contour interactions offer no explanations for this effect. Yet it is entirely consistent with the object substitution theory as described in the text. This can be seen in Demonstration 1 on the Internet site.

Displays were presented on an Applevision monitor controlled by a Macintosh computer running VScope software (Enns \& Rensink, 1992). The background screen was set to mid-gray (50\% lightness using the Macintosh HSL color picker), and the stimuli were drawn in black (0\% lightness using HSL). Observers sat with their heads on a chinrest, $50 \mathrm{~cm}$ from the screen. A central small fixation cross was continuously visible on each trial, and observers were instructed to fixate it throughout the trial.

On each trial, two standing illusion displays were presented, one to each side of fixation in Experiments 1, 3, and 4, and one above and below the fixation in Experiment 2. Observers were told that the displays were the same on each side of fixation and that there were two identical displays because we were interested in the visibility of the bars in the parafovea (Macknik \& Livingstone, 1998). Observers were also told that if their eyes moved from the fixation cross during a trial, they should refixate before judging the visibility of the central bars. Thus, before they judged the visibility of target shapes in each display, the cycling displays had been on for at least 1-2 sec. The observer was also repeatedly reminded throughout the testing session to fixate on the central cross before determining the final visibility rating for the target shape in any given display.

Each observer was tested on nine trials in each condition of an experiment, with conditions randomly interleaved within an experiment. This generated a total of 108 observations for each data point in the graphs. Data were analyzed by a repeated measures analysis of variance (ANOVA), and the statistical significance of all reported effects was at least $p<.05$.

\section{EXPERIMENT 1 No Psychophysical Evidence for Termination Asynchrony}

The first experiment explored a wider range of temporal conditions in the standing wave illusion than have been tested previously (Macknik \& Livingstone, 1998; Macknik et al., 2000). This was an important first step because the termination asynchrony hypothesis was based on experiments involving only two display frames (target and mask bars) and on a relatively restricted range of frame durations (20-90 msec) (Macknik \& Livingstone, 1998).

In Experiment 1 the standing wave illusion was examined for a single target bar flickering in counterphase with two flanking masking bars (Figure 1). The display frames are numbered " 1 " and " 2 ," but it is important to bear in mind that these displays are cycling continuously while they are being viewed. Thus, the initial order of presentation of the two frames has no bearing on the appearance of the displays or on the results obtained.

The duration of the target bar ranged from $17 \mathrm{msec}$ to $200 \mathrm{msec}$ and the duration of the flanking bars ranged from $17 \mathrm{msec}$ to $1,000 \mathrm{msec}$. The interstimulus interval (ISI) between target and flanking bars was always 0 msec. Each bar subtended $3.0^{\circ} \times 0.6^{\circ}$, and each standing wave display was centered $3^{\circ}$ from fixation.

Whereas termination asynchrony theory predicts that masking is maximal when the target and mask terminate with an asynchrony near $100 \mathrm{msec}$, object substitution theory holds that masking will become asymptotically stronger as mask duration is increased (Di Lollo et al., 2000; Enns 


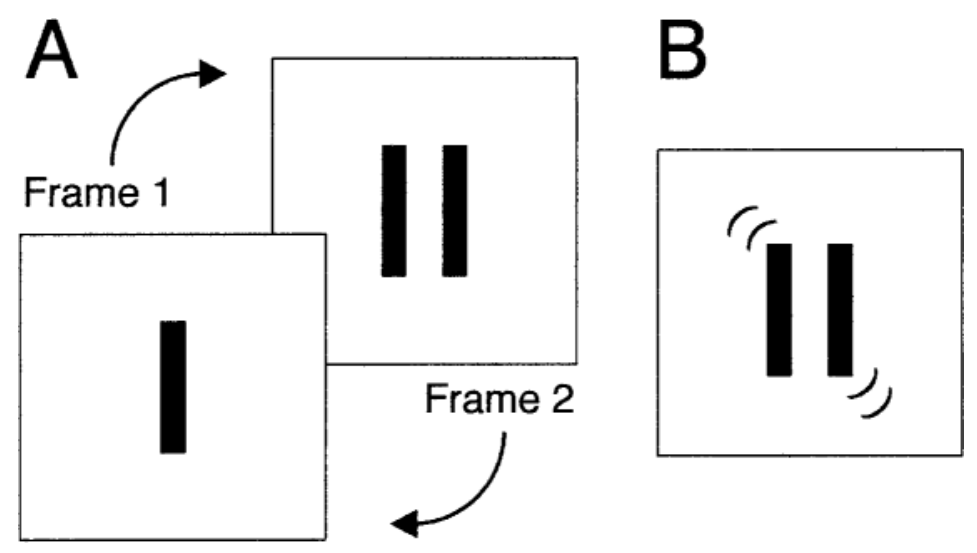

Figure 1. (A) Display sequence for the standing wave illusion. (B) The appearance of the display when the two frames are alternating at 50-150 msec per frame is of two dancing bars separated by a space.

\& Di Lollo, 2000). Indeed, object substitution holds no special role for stimulus transients, so that masking is predicted even when there is no asynchrony in stimulus onsets and there is no mask offset (Di Lollo et al., 2000; Enns \& Di Lollo, 2000).

The results shown in Figure 2A support object substitution. Masking was similar for all target durations and increased monotonically as the duration of the flanking bars was increased. If anything, the data indicate that there was even more masking for longer target than shorter target durations, but this difference was not significant. The visibility data for mask durations beyond $217 \mathrm{msec}$ are not shown because they were equal to or even lower at longer durations. There was no hint of a termination asynchrony effect for any target or mask duration.

The possible criticism that visibility ratings may not be sufficiently sensitive to reveal effects of termination asynchrony is addressed in Figure 2B. Shown are the mean visibility ratings for a target duration of $100 \mathrm{msec}$, as a function of varying separations between target and flanking bars. Very small spatial separations had a reliable influence on the ratings of all observers $(p<.01)$, suggesting that there was every opportunity for temporal variables to reveal similar sensitivity.

One surprising aspect of the data in Figure 2A is that significant masking occurred in many conditions when flanking bars were flickering for much shorter durations than target bars. For example, a target flickering for 200msec durations was masked robustly by flanking bars of only $83-\mathrm{msec}$ and $117-\mathrm{msec}$ duration. The fact that targets bars can be made invisible by flanking bars of only half their duration strongly suggests that nonlocal spatiotemporal factors are at work.

\section{EXPERIMENT 2 Nonlocal Influences on Masking}

Experiment 2 tested for nonlocal masking effects by comparing the three conditions shown in Figure 3. Fig- ure $3 \mathrm{~A}$ shows the standard condition in which Frame 1 was set to a constant duration of $100 \mathrm{msec}$ and Frame 2 was presented for either 17 or $100 \mathrm{msec}$. The other two conditions were identical, with the exception that an additional bar was added to each frame in Figure 3B, and two bars were added to each frame in Figure 3C. The displays were centered and positioned above and below the fixation point instead of on either side of fixation, as in the previous experiment. This was done to permit the perceptual judgment of the visibility of the central bar to be as accurate as possible. Each bar in these displays was $1.5^{\circ} \times$ $0.3^{\circ}$, and the upper and lower sets of bars in each display were centered $1.5^{\circ}$ from fixation.

Although these displays were identical with regard to the central three bars in each condition, very different patterns of masking were observed, as shown in the middle panel of Figure 3. In Condition 3A, the central bar was relatively visible when the flanking bars were presented for only $17 \mathrm{msec}$, but became much less visible when the flanking bars were shown for $100 \mathrm{msec}$ on each cycle. This is the same result reported in Experiment 1 for a central bar of $100 \mathrm{msec}$ alternating with two flanking bars. In Condition 3B, an alternation sequence of $100 \mathrm{msec}$ for both frames resulted in a highly visible central bar. Note that here the central bar was accompanied in the same frame by two other bars that were now the outermost flankers. In Condition 3C, the visibility of the central bar was again reduced when each frame was shown for $100 \mathrm{msec}$. Notably, the central bar in this condition appeared in a frame where all bars were flanked on both sides by bars in the alternating frame.

This pattern of masking was assessed in a second way by having 10 independent observers view the same displays, this time indicating the total number of "darker bars" they could see on each trial. Observers were told that the displays above and below fixation were identical and that their task was to count the number of apparently darker bars in either one of these displays, while keeping their eyes on the fixation cross. It was expected that when 

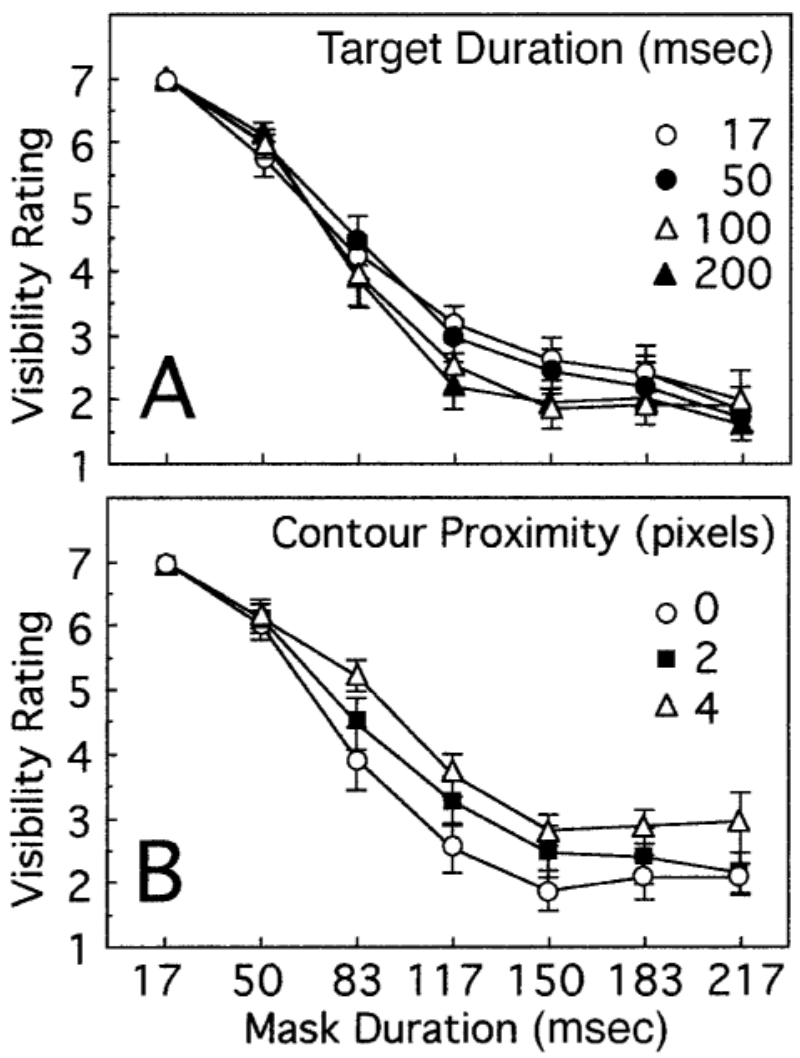

Figure 2. (A) Mean rated visibility of the central target bar for various combinations of target and flanking bar durations in Experiment 1. Error bars represent $1 S E$. Data are shown for only the nearest target-flanker contour proximity (0-pixel separation). Separations of 2 and 4 pixels ( 4 and 8 min of arc) were also tested, but these are not shown for all target durations. (B) Mean rated visibility of a 100-msec target as a function of both mask duration and flanking contour proximity. Error bars represent $1 S E$.

Frame 1 was shown for $100 \mathrm{msec}$ and Frame 2 for $17 \mathrm{msec}$ that the number of bars in Frame 1 would be the basis for the response. The main question was therefore how many bars would be seen when each of the frames was shown for an equal $100 \mathrm{msec}$ on each cycle.

The results, shown in the lower panel of Figure 3, were unequivocal. The number of bars in Frame 1 of each display formed the basis for the response when it was the longer duration frame, but the number of bars in Frame 2 determined the response when the frames were equal in duration. This means that whether the central bar was visible or masked depended critically on whether it appeared in the same frame as the outermost flanking bars. When it appeared with these visible bars, the central bar was itself visible; when it appeared with other bars that were masked, it too was masked. Masking of the central bar was clearly not being determined principally by local edge interactions.

These results point to two important features of standing wave masking. First, there is a competition for the edge shared by bars in the two alternating frames. This competition is resolved in favor of the bar that is most visible on both temporal and spatial grounds. If there is a large temporal imbalance between the durations of the two video frames, the bars of longer duration will be more visible. However, if the edges of some bars are not flanked, and therefore not in any competition for a common edge, those bars will be seen in favor of bars that are flanked on two sides. As seen in Experiment 1, these geometric considerations permit even flankers of relatively brief duration to mask central bars of longer duration.

Second, there appears to be a cooperative process in which the visibility of any given bar depends on whether
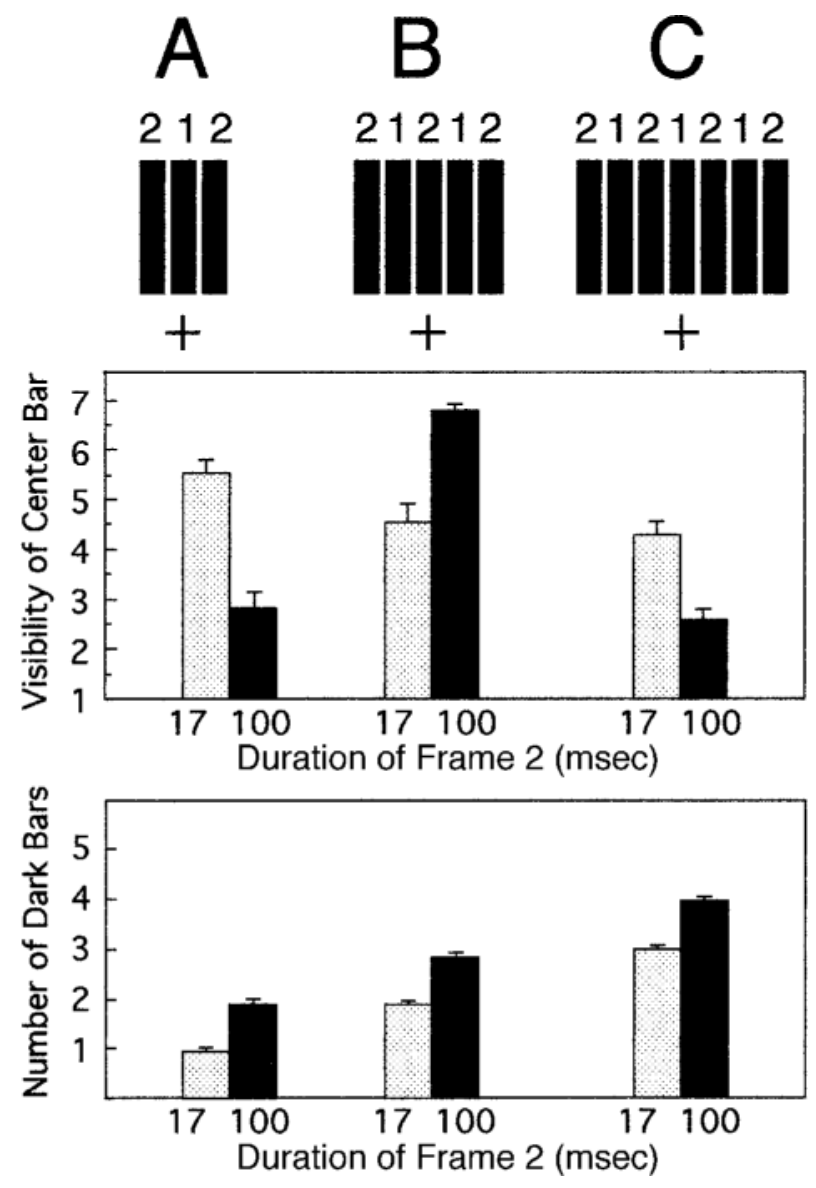

Figure 3. The three conditions tested in Experiment 2. The digits above each of the shapes refer to the frames in which these shapes appeared. The data shown in the middle panel are mean visibility ratings of the central target bar for the two mask durations. Error bars represent $1 S E$. Masking is indicated by the reduced visibility of the central target bar $(100-\mathrm{msec}$ duration $)$ in Conditions $A$ and $C$ when the duration of Frame 2 is also $100 \mathrm{msec}$. The 17-msec duration was included for referencing the ratings to conditions where no masking occurred. The data shown in the lower panel represent the mean number of "darker" bars reported by a separate group of participants viewing the same displays. When the duration of Frame 2 was $17 \mathrm{msec}$, all participants saw the bars in Frame 1 as darker, as is expected given their longer duration $(100 \mathrm{msec})$. But when the duration of Frame 2 was $100 \mathrm{msec}$, the number of darker bars matched those in Frame 2, even though the Frame 1 bars were presented for a similar duration. Error bars represent $1 S E$. 
other bars in the same video frame are relatively visible. If the central bar in this experiment was shown in the same frame as other bars that were visible, either because those other bars were shown for a relatively longer duration or because some of them were not flanked on both sides by other bars, then the central bar was itself visible. If the central bar was shown in the same frame with other bars that were masked, it too was masked, even though its local spatiotemporal characteristics had not changed at all. These cooperative influences clearly extend beyond the range of the visual units required to register the individual edges of a bar.

\section{EXPERIMENT 3 Shape Similarity Influences Masking}

Experiment 3 examined the role of shape similarity in the standing wave illusion. Previous demonstrations have all been based on identical target and flanker shapes that share large portions of common contours (Macknik \& Livingstone, 1998; Macknik et al., 2000). In one exception, but again involving only two-frame displays, target bars in Frame 1 were spatially overlaid by masking bars in Frame 2 of varying width (but not length) (Macknik et al., 2000). Counterintuitively, masking was strongest for shapes that were most similar to the targets, not by masks that were much larger and therefore had greater energy. The authors interpreted this as support for the spatially local nature of masking, since the nearest edges yielded the strongest masking.

Object substitution predicts the same result but for a different reason. Flanking shapes that are similar to target shapes mask most effectively because the visual attributes of a target that have not yet been fully identified can be incorporated most easily into a visible flanking shape that is similar. Such an illusory conjunction (Treisman \& Gelade, 1980), or feature inheritance effect (Herzog \& Koch, 2001; Wilson \& Johnson, 1985), permits many of the properties of the masked shape to be seen, but they are no longer bound correctly to their actual spatiotemporal location. This is the sense in which the shape in the target location becomes "invisible." When too many of the properties of the target and mask are inconsistent with one another, the reentrant processes have a harder time reconciling the patterns activated by each of these shapes and feature inheritance is less likely to occur. Instead, both the target and the flanking shapes are seen. This phenomenon has been coined "shine through" (Herzog, Fahle, \& Koch, 2001; Herzog, Koch, \& Fahle, 2001), referring simply to the failure of masking by the flanking or overlaid shapes.

The shapes tested in Experiment 3 pitted the influence of shared local contours against global shape similarity (Figure 4). They included (1) the standard vertical bars, subtending $1.5^{\circ} \times 0.4^{\circ}$; (2) the same vertical target bar flanked by much wider bars, subtending $1.5^{\circ} \times 1.2^{\circ}$; (3) bars aligned horizontally, subtending $0.4^{\circ} \times 1.2^{\circ}$, so that only their short ends were abutting; and (4) the same horizontal bar abutted by much taller flanking bars, sub-
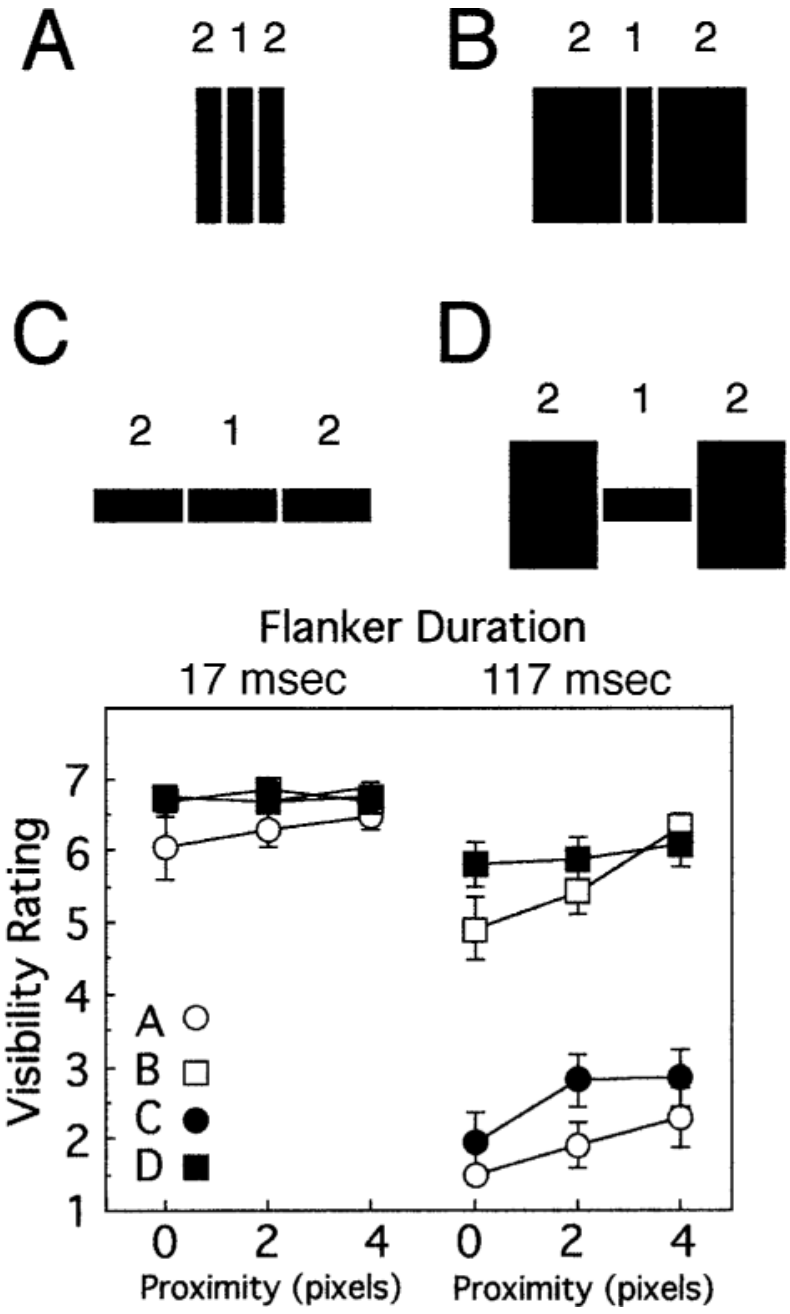

Figure 4. The four conditions tested in Experiment 3 (A-D). The digits above each of the shapes refer to the frames in which these shapes appeared. The data shown are mean visibility ratings of the central target shapes for two mask durations in Experiment 3. Error bars represent $1 S E$. Masking is evidenced by the reduced visibility of the target shape when the mask duration is $117 \mathrm{msec}$. The data for mask durations of $17 \mathrm{msec}$ are included for referencing purposes.

tending $1.5^{\circ} \times 1.2^{\circ}$. As in Experiment 1, each display was centered $3^{\circ}$ from fixation on the left and the right.

Any theory based on local edge interactions would predict similar, strong masking in Conditions $\mathrm{A}$ and $\mathrm{B}$, where the target contour is almost entirely surrounded. There should also be little, if any, masking in Conditions $\mathrm{C}$ and $\mathrm{D}$, where little of the target contour is surrounded. Object substitution theory, on the other hand, predicts maximum masking effects in Conditions $\mathrm{A}$ and $\mathrm{C}$, where the shapes are similar, and little masking in Conditions $\mathrm{B}$ and $\mathrm{D}$, where many of the contours are flanked but the shapes differ.

The results in Figure 4 are decisively in favor of object substitution. Standing wave masking is predicted by similarity of the target and flanking shapes, not by the extent 

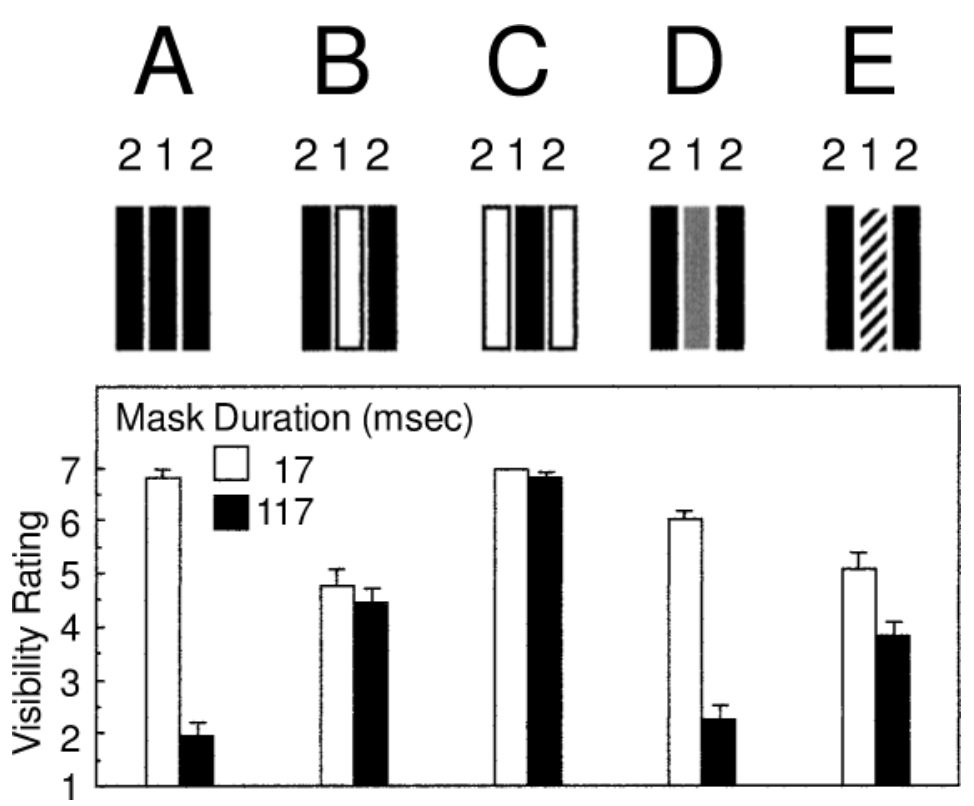

Figure 5. The five conditions tested in Experiment 4 (A-E). The lower panel shows the mean rated visibility of the central target when mask duration was either $17 \mathrm{msec}$ (reference visibility) or $117 \mathrm{msec}$ (potential masking). Error bars represent $1 S E$.

to which the target shape is surrounded by abutting contours. These effects of shape similarity indicate that a complete account of standing wave masking must incorporate a level of processing beyond that of local contour interactions.

\section{EXPERIMENT 4 Surface Relations Influence Masking}

Experiment 4 explored the role of surfaces in the standing wave illusion. Since global shape is important in the illusion, as already shown, perhaps other nonlocal visual properties are also important. Although accounts based on local contour interactions would not predict such effects, object substitution theory suggests them quite naturally. If higher level shape and pattern processes are used to bind specific contours in the visible percept, then other properties of shape analyzed by extrastriate visual regions, such as lightness, color, and texture, might be used as well.

The surface relations tested are shown in Figure 5 and include (1) the standard vertical bars, (2) an outline vertical target bar flanked by two solid bars, (3) a solid target bar flanked by two outline bars, (4) a medium-gray target bar flanked by two black bars, and (5) a striped vertical bar flanked by two black bars. Each vertical bar subtended $1.5^{\circ} \times 0.3^{\circ}$, and each standing wave display was again centered $3^{\circ}$ from fixation (Figure 5).

The comparison of masking between solid shapes (Con$\operatorname{dition} \mathrm{A}$ ) and outline shapes (Conditions B and C) pits the importance of local edges (which are similar in both cases) against the importance of surface color (which is maximally different without involving a reversal in contrast polarity). The medium-gray target (Condition D) tests an intermediate point between these extremes, whereas the striped target bars (Condition E) test whether the texture internal to the target shape is important over and above the issues of average lightness (which are the same in Conditions D and E).

Standing wave masking was indeed influenced by the surface properties of target and mask shapes (Figure 5). Relative to the standard Condition A, there was very little masking in Condition B, even though the outline target bar had less overall energy than the solid flanking bars and was generally not rated to be as visible as the black bar in Condition A. Any argument that this outcome reflects the advantage of two-edged outlines in the target is challenged by Condition $\mathrm{C}$, where no masking was again in evidence, even though the flanking bars now contained the two-edged outlines.

Condition D, in which the lightness of the target bar was intermediate to these two extremes, pointed to an additional feature of standing wave masking. As can be seen in Demonstration 3 on the Internet site, and as reported spontaneously by observers, the medium-gray target bar, although masked quite well by the flanking bars, also gave the flanking bars a "smudgy gray" appearance. This is another example of the illusory conjunctions of feature properties and location described earlier for target length (General Method and Demonstration 1).

Finally, the striped target in Condition E was not masked as effectively as the gray target without stripes in Condition D, even though their average lightness was the same. 
Observers reported that the stripes were often seen in the center of the display (where they actually were), but occasionally observers reported seeing the stripes superimposed on the flanking bars. These results argue strongly in favor of standing wave masking being determined by surface properties in addition to shape properties.

\section{GENERAL DISCUSSION}

The standing wave illusion involves continuously visible percepts that are not veridical. Shapes in alternating videoframes that are displayed for equal amounts of time can either be visible or invisible, depending on spatial, temporal, and featural factors. Furthermore, properties of the shapes that are not seen are sometimes seen clearly, albeit in incorrect locations, as belonging to the shapes that are seen. Importantly, this illusion is not simply a repetitive form of classical metacontrast or another previously reported instance of backward masking. The hallmark of those forms of masking is that, when shapes compete for the same local edge, temporally leading shapes are less visible than temporally trailing ones (Enns \& Di Lollo, 2000). In the standing wave illusion, the same local edges are involved when the shapes in either one or the other frame are seen. Because these displays cycle continuously, temporal order is ruled out as the determinant of visibility. This argues against low-level explanations based on competition between magno- and parvocellular pathways or time-weighted averaging (Breitmeyer, 1984).

Instead, the standing wave illusion provides rich information about the early stages of object formation and feature binding in the visual system. The most important demonstration of the present study is that standing wave masking is not dependent primarily on either local edge interactions (Experiment 2) or the amount of contour in the central shape that is flanked by the bars in the alternate frame, but rather on the similarity in shape (Experiment 3 ) and surface (Experiment 4) between the bars in the two frames.

This conclusion does not deny the relevance of the local contour interactions, whose importance can be seen in the neurophysiological data derived from masking of sameshape stimuli in cycling displays (Macknik \& Haglund, 1999; Macknik \& Livingstone, 1998; Macknik et al., 2000). Their importance can also be seen in the present study, where contour proximity between the central and flanking bars was a determining factor in the amount of masking that occurred (see Figure 2B for these data in Experiment 1 and Figure 4 for similar data in Experiment 3 ). Yet, the present data also show that the amount of flanking contour is of minimal importance in predicting masking when nonlocal factors are at work (Figure 3 ) and different shapes and surfaces are involved (Figures 4 and 5). Therefore, a full account of the data requires an explanation of both the important role of shape and surface similarity and the importance of local contour proximity in determining masking.

Object substitution theory proposes that this comes about through interactions between higher level units (e.g., the shape-, pattern-, and texture-sensitive units of extrastriate cortical regions) and lower level units (e.g., contour-sensitive units in Area V1). Specifically, the lowlevel units are entrained by reentrant signals from higher level units, and it is this recurrent processing that forms the basis of the conscious percept (Hupe, James, Payne, Lomber, Girard, \& Bullier, 1998; Lamme \& Roelfsema, 2000; Sillito, Jones, Gerstein, \& West, 1994).

Object substitution theory, by virtue of its proposed reentrant connections between lower and higher levels of processing, provides a useful framework for studying these dynamic interactions. But this is only a beginning. The qualitative predictions that can be derived from object substitution theory in its current form (e.g., mask duration is more critical than the specific timing of target and flanker transients, nonlocal spatial and temporal factors are important, and shape and surface variables are important) must in future give way to more specific predictions about which visual attributes will exert their influence most quickly and over what spatial range the nonlocal effects will operate. The details of shape and surface similarity that are relevant to masking must also be worked out. These findings will likely come from studies involving a combination of neuroanatomical methods (e.g., lesioning or cooling of specific extrastriate regions in conjunction with multisite electrophysiology), cortical stimulation (e.g., transcranial magnetic stimulation), brain imaging (e.g., event-related potentials and magnoencephalograhy), and behavior (e.g., psychophysics involving carefully controlled visual properties and behavioral measures that are based both on phenomenology and performance).

\section{REFERENCES}

BreITMEYer, B. G. (1984). Visual masking: An integrative approach. New York: Oxford University Press.

Di Lollo, V., Enns, J. T., \& Rensink, R. A. (2000). Competition for consciousness among visual events: The psychophysics of reentrant visual processes. Journal of Experimental Psychology: General, 129, 481-507.

ENns, J. T., \& Di Lollo, V. (2000). What's new in visual masking? Trends in Cognitive Sciences, 4, 345-352.

ENNS, J. T., \& RENSINK, R. (1992). VScope(tm): Vision testing software for the Macintosh. Vancouver: Micropsych Software.

Herzog, M. H., Fahle, M., \& Koch, C. (2001). Spatial aspects of object formation revealed by a new illusion, shine-through. Vision Research, 41, 2325-2335.

Herzog, M. H., \& Koch, C. (2001). Seeing properties of an invisible object: Feature inheritance and shine-through. Proceedings of the $\mathrm{Na}$ tional Academy of Sciences, 98, 4271-4275.

Herzog, M. H., Кoch, C., \& Fahle, M. (2001). Shine-through:Temporal aspects. Vision Research, 41, 2337-2346.

Hupe, J. M., James, A. C., Payne, B. R., Lomber, S. G., Girard, P., \& BULLIER, J. (1998). Cortical feedback improves discrimination between figure and ground by V1, V2, and V3 neurons. Nature, 394, 784-787.

Lamme, V. A. F., \& Roelfsema, P. R. (2000). The distinct modes of vision offered by feedforward and recurrent processing. Trends in $\mathrm{Neu}$ rosciences, 23, 571-579.

Macknik, S. L., \& Haglund, M. M. (1999). Optical images of visible and invisible percepts in the primary visual cortex of primates. Proceedings of the National Academy of Sciences, 96, 15208-15210.

MackniK, S. L., \& Livingstone, M. S. (1998). Neuronal correlates of 
visibility and invisibility in the primate visual system. Nature Neuroscience, 1, 144-149.

Macknik, S. L., Martinez-Conde, S., \& Haglund, M. M. (2000). The role of spatiotemporal edges in visibility and visual masking. Proceedings of the National Academy of Sciences, 97, 7556-7560.

Sillito, A. M., Jones, H. E., Gerstein, G. L., \& West, D. C. (1994). Feature-linked synchronization of thalamic relay cell firing induced by feedback from the visual cortex. Nature, 369, 479-482.
Treisman, A., \& Gelade, G. (1980). A feature integration theory of attention. Cognitive Psychology, 12, 97-136.

Wilson, A. E., \& Johnson, R. M. (1985). Transposition in backward masking: The case of the travelling gap. Vision Research, 25, 283-288.

(Manuscript received March 2, 2001;

revision accepted for publication September 21, 2001.) 